\title{
Putidaredoxin Binds to the Same Site on Cytochrome P450cam in the Open and Closed Conformation
}

Shu-Hao Liou ${ }^{\mathbf{a}, \mathbf{b}}$, William K. Myers ${ }^{\mathbf{c}}$, Jason D. Oswald ${ }^{\mathbf{a}}$, R. David Britt ${ }^{\mathbf{a}}$ and David B. Goodin ${ }^{\mathbf{a}^{*}}$

${ }^{a}$ Department of Chemistry, University of California, Davis, CA 95616, United States

${ }^{\mathrm{b}}$ Research Group EPR Spectroscopy, Max-Planck-Institute for Biophysical Chemistry, Göttingen 37077, Germany

${ }^{c}$ Centre for Advanced Electron Spin Resonance, Inorganic Chemistry Laboratory, University of Oxford, Oxford OX1 3QR, United Kingdom

*Corresponding author, email address: dbgoodin@ucdavis.edu 


\begin{abstract}
Cytochrome P450 CYP101A1 (P450cam) hydroxylates camphor by receiving two distinct electrons from its unique reductase, putidaredoxin $(\mathrm{Pdx})$. Upon binding ferric $\mathrm{P} 450$ cam, $\mathrm{Pdx}$ is now known to trigger a conformational change in the enzyme. This Pdx-induced conversion may provide the trigger to coordinate enzyme turnover and protect the enzyme from oxidative damage, so the interactions responsible for this conversion are of significant current interest. This proposed role for Pdx requires that its interactions with P450cam are different for the open and closed conformations. In this study, we show that the binding thermodynamics of Pdx does indeed differ in the predicted way when the conformation of P450cam is held in different states. However, Double Electron-Electron Resonance (DEER) measurements of intermolecular distances in the Pdx/P450cam complex show that the geometry of the complex is nearly identical for the open and closed states of P450cam. These studies show that Pdx appears to make a single distinct interaction with its binding site on the enzyme, and triggers the conformational change through very subtle structural interactions.
\end{abstract}




\section{INTRODUCTION}

Cytochromes P450 forms a superfamily of heme containing monooxygenases with well over 20,000 members, each sharing a similar protein architecture and function to carry-out C-H bond activation and hydroxylation. ${ }^{1-3}$ The enzyme utilizes both $\mathrm{O}_{2}$ and a source of electrons to carryout oxidation of substrate. The cycle begins when substrate binds near the distal heme face of ferric P450 and causes a shift of the heme from a low-spin to high-spin state. This transition increases the reduction potential of $\mathrm{P} 450$ and promotes electron transfer (ET) from the reductase to generate the ferrous state and allow for $\mathrm{O}_{2}$ binding. Further proton and electron transfer to the ferrous- $\mathrm{O}_{2}$ form generates the ferric-hydroperoxo complex ${ }^{4-7}$ Heterolytic cleavage of the peroxy bond produces a highly reactive intermediate, Compound I, which is responsible for oxygen insertion into the substrate by hydrogen atom abstraction and radical rebound. ${ }^{7-11}$ Cytochrome P450cam (CYP101) from Pseudomonas putida is the model enzyme for the P450 superfamily. ${ }^{12-}$ ${ }^{14}$ It carries out the conversion of camphor to 5-exo-hydroxycamphor under conditions where the bacterium uses camphor as the primary carbon source for growth. The enzyme is highly specific for its reductase, with an absolute requirement for the native putidaredoxin $(\operatorname{Pdx})$ to provide the second ET. ${ }^{15}$ This may contribute to the highly coupled behavior of P450cam in which electrons and $\mathrm{O}_{2}$ are incorporated into substrate with high efficiency.

Pdx has long been known to play an effector role in binding to P450cam by inducing spectroscopic changes that have structural impact on proximal and distal sites of the enzyme. ${ }^{15-20}$ The exact nature of the effector role has remained unknown, but recent insights have been obtained through nearly simultaneous reports of the crystal structure of the tethered P450cam/Pdx complex ${ }^{21}$ and solution studies by Double Electron-Electron Resonance (DEER). ${ }^{22}$ Both of these studies showed that binding of Pdx to the closed, substrate-bound ferric enzyme 
caused its conversion to the open state ${ }^{23-25}$. This result was initially controversial, as an NMR study did not support this conversion to the open state. ${ }^{26,27}$ However, subsequent DEER observations at alternative labeling sites on the enzyme have shown that Pdx clearly induces the structural conversion depending on the position and nature of the labeling. ${ }^{28}$ An important observation that will be of particular use in the current study is that the Pdx-induced conformational change was not observed when reduced $\mathrm{Pdx}\left(\mathrm{Pdx}_{\mathrm{re}}\right)$ was bound to the ferrous-CO state of camphor-bound P450cam $\left(\mathrm{P} 450 \mathrm{cam}\left(\mathrm{Fe}^{2+} \mathrm{CO} \cdot \mathrm{S}\right)\right) .{ }^{22}$ A subsequent DEER study has shown that the mixed oxidation state, with $\mathrm{P} 450$ cam $\left(\mathrm{Fe}^{2+} \mathrm{CO} \cdot \mathrm{S}\right)$ bound to oxidized $\mathrm{Pdx}$ does not undergo the closed to open transition. ${ }^{28}$ Thus, the effector role of Pdx on P450cam appear to be narrowed to two possibilities: either Pdx binding induces a conversion of P450cam to the open state and this conversion is negated upon $\mathrm{CO}$ binding, or $\mathrm{Pdx}$ induces differing structural changes on P450cam depending on its oxidation state.

Regardless of the details of the effector role of Pdx, it is not understood how Pdx binding to a site on one side of the enzyme, near the proximal heme ligand, can affect peroxy bond cleavage on the opposite side, within the distal substrate binding channel. In one view, ${ }^{21,28,29}$ a small movement of the $\mathrm{C}$ helix near the $\mathrm{Pdx}$ binding site, is transmitted through interactions in the I helix to enable opening of the $\mathrm{F}$ and $\mathrm{G}$ helices. This opening may result in an alteration of the hydrogen bonding interactions between Gly248 and Thr252 of the I helix, as seen in a large number of tethered substrates that induce the open conformation. ${ }^{25}$ In concert with this, $\mathrm{O}_{2}$ binding itself may push the I helix toward a more open state, resulting in the occupation of a catalytic water molecule above the distal heme face ${ }^{25}$ that is proposed to protonate the distal peroxy bond cleavage. ${ }^{7}$ It has been proposed that this conversion is the trigger for Compound I formation and that substrate turnover occurs as the enzyme moves to the open state, perhaps at 
an intermediate conformation similar to that previously trapped by binding of the synthetic tethered substrate analog Ada-EG-Dans. ${ }^{25}$ This proposal implies that the binding interaction between Pdx and P450cam is different in the open and closed conformations and suggests that Pdx binds to the open conformation more strongly, at least enough so to drive the structural transition. In this paper, we directly examine the energetics of the interaction of $\mathrm{Pdx}$ with P450cam in different conformational states. We also use DEER measurements in which pairs of spin-labels are placed across the interface between Pdx and P450cam, to observe how similar the complex interface is in the open and closed conformations. The results are discussed in terms of the rapidly developing understanding of the effector role of Pdx in P450cam catalysis. 


\section{METHODS}

Site-directed mutagenesis, expression and purification of proteins. DNA mutation and protein purification procedures of $\mathrm{P} 450$ cam were followed by previous publications. ${ }^{24,28}$ To prepare the P450cam mutant (P450cam-4S) without surface exposed cysteines, four cysteines (C58, C85, C136 and C285) were mutated to serines. ${ }^{22,}{ }^{24}$ Four spin-labeling sites on P450cam were constructed by introducing an additional exposed cysteine into the P450cam-4S background at positions S48C, S190C, Q227C or Q272C by site-directed mutagenesis. For wild-type Pdx, two cysteines (C73 and C85) are surface exposed, while four others (C39, C45, C48 and C86) are coordinated to the iron-sulfur cluster $([2 \mathrm{Fe}-2 \mathrm{~S}]) .{ }^{30} \mathrm{C} 85 \mathrm{~S} / \mathrm{C} 73 \mathrm{~S}$ and C85S pET-Pdx plasmid was kindly provided by Prof. Thomas Poulos (UC Irvine). C85S pET-Pdx was used as one of the singly exposed cysteine (C73) species for labeling Pdx. Two alternative sites at S29C or E14C were introduced into the C85S/C73S background by site-directed mutagenesis. For Pdx expression, the pET-Pdx plasmid was transformed into BL21 (DE3) of E. Coli. Cells were grown in $17 \times 100 \mathrm{~mm}$ culture tubes with $\mathrm{LB}$ broth containing ampicillin $(100 \mathrm{mg} / \mathrm{ml})$ for 8 hours at 37 ${ }^{\circ} \mathrm{C}$, and transferred to TB (terrific broth) broth containing ampicillin $(100 \mathrm{mg} / \mathrm{ml})$ at $37{ }^{\circ} \mathrm{C}$ for 12 hours. After cells were induced by $0.4 \mathrm{mM}$ isopropyl $\beta$-D-1-thiogalactopyranoside (IPTG), the temperature was dropped to $30{ }^{\circ} \mathrm{C}$ for 24 hours. Cells were suspended in $50 \mathrm{mM}$ potassium phosphate (pH 7.5) before being lysed by a French press. After centrifugation to remove the cell debris, the cell extract was loaded into a $60 \mathrm{ml}$ DEAE anion exchange column (DEAE Sepharose CL-6B, GE Healthcare), the column was washed with $0-100 \mathrm{mM} \mathrm{KCl}$ and protein was eluted at $150 \mathrm{mM} \mathrm{KCl}$. The protein fractions with $\mathrm{A}_{412} / \mathrm{A}_{280}>0.1$ ratio were collected and loaded onto a 1.8 L Sephacryl S-200 gel-filtration column. The fraction with $\mathrm{A}_{412} / \mathrm{A}_{280}>0.48$ was collected for later experiments. 
Isothermal titration calorimetry. ITC data was collected using a Micro Calorimetry System Isothermal Calorimeter (MicroCal Inc.) at $25{ }^{\circ} \mathrm{C} . \mathrm{Pdx}(1.5 \mathrm{mM})$ was titrated into $50 \mu \mathrm{M}$ P450cam in $50 \mathrm{mM}$ Tris and $150 \mathrm{mM} \mathrm{KCl}(\mathrm{pH}$ 7.4). Additional ligand concentrations were 1 $\mathrm{mM}$ metyrapone, $1 \mathrm{mM}$ camphor and $50 \mu \mathrm{M}$ Ada-Eg-Dans. Both P450cam and Pdx were prepared for titration by buffer exchange using PD-10 (GE Healthcare) columns. The sample cell was stirred continuously with a rotary syringe at $400 \mathrm{rpm}$. Data analysis was carried out using Origin software, and reported errors are the standard deviation of multiple measurements. After initial baseline subtraction and integration, the data was fitted to a single binding site model. The synthetic ligand Ada-Eg-Dans, containing an adamantane coupled through an ethylene glycol linker to a dansyl group has been previously described (referred to as AdaC1-Etg-Dans) and binds to P450cam to produce a conformation that is intermediate between open and closed (PDB entry 3P6O). ${ }^{25}$

DEER Sample Preparation. Prior to spin-labeling of P450cam, DTT and camphor were removed using two sequential PD-10 gel-filtration columns (GE Healthcare) as previously described $^{24}$. Site-directed spin-labeling was performed by using 150 mM MTSL ((1-oxyl-2,2,5,5tetramethyl- $\Delta^{3}$-pyrroline-3-methyl) methanethiosulfonate, Toronto Research Chemicals) dissolved in DMSO. For P450cam, a 10 fold molar excess of MTSL was used in the labeling reactions for 10 minutes as previously described.$^{24}$ For Pdx, the labeling reaction consisted of a two-fold stoichiometric excess of MTSL, and it was determined that three minutes incubation at room temperature was sufficient for labeling of the surface cysteines before disrupting the four cysteines (C39, C45, C48 and C86) coordinated to the iron-sulfur cluster (supporting information). Excess MTSL was removed by $6 \times 5$ fold repeated dilutions by ultrafiltration (Amicon ultracentrifugation, $10 \mathrm{kDa}$ cutoff for $\mathrm{Pdx}$ and $30 \mathrm{kDa}$ cutoff for P450cam, 
respectively) and the buffer was exchanged into $99 \% \mathrm{D}_{2} \mathrm{O}$ and $50 \mathrm{mM}$ Bis-Tris (pD 7.6). For the reduced samples, $1 \mathrm{mM}$ camphor and $150 \mathrm{mM} \mathrm{KCl}$ was added into the same buffer. The extent of labeling was examined by Mass Spectrometry (ESI-MS) with an Agilent 1260/6120 series LC/MS (6000 V capillary voltage). The fragmentation voltage was $150 \mathrm{~V}$ for P450cam and $80 \mathrm{~V}$ for Pdx. A 4.6×50 mm Poroshell C18 column (Agilent) with a gradient of 30-80 \% acetonitrile in water containing $0.1 \%$ formic acid was used to remove salt from P450cam and Pdx prior to ESIMS. For oxidized P450cam $\left(\mathrm{Fe}^{3+}\right) / \mathrm{Pdx}_{\text {ox }}$ DEER sample, $150 \mu \mathrm{M}$ P450cam was mixed with 150 $\mu \mathrm{M}$ Pdx in $30 \% \mathrm{~d}_{8}$-glycerol (Cambridge Isotope Laboratories) as a cryogenic glassing agent, 15 $\mu 1$ of the mixture was loaded into a quartz tube $(100 \mathrm{~mm}$ length, $1.1 \mathrm{~mm}$ I.D. and $1.6 \mathrm{~mm}$ O.D., VitroCom) and then flash-frozen into liquid nitrogen. For the $\mathrm{P} 450 \mathrm{cam}\left(\mathrm{Fe}^{2+} \mathrm{CO} \cdot \mathrm{S}\right) / \mathrm{Pdx}$ re complex, $150 \mu \mathrm{M}$ P450cam, $150 \mu \mathrm{M} \mathrm{Pdx}, 3 \mu \mathrm{M}$ Pdr and $30 \% \mathrm{~d}_{8}$-glycerol was mixed in anaerobic environment with $30 \mathrm{mM}$ NADH before purging with carbon monoxide for more than five minutes and then flash frozen in liquid nitrogen.

EPR and DEER measurements. Continuous-wave EPR (cwEPR) measurements were performed using Elexsys-II E500 spectrometer (X-band) with a Bruker SHQE resonator (ER4122SHQE) and an Oxford liquid helium cooling system. All DEER measurements were performed on a Bruker Elexsys E580 spectrometer with a Bruker EN5107D2 Q-band EPR/ENDOR probe head at approximately $34 \mathrm{GHz}$ and $30 \mathrm{~K}$. For DEER experiments, a fourpulse sequence $\left(\frac{\pi^{*}}{2}-\tau_{1}-\pi^{*}-\left(\tau_{1}+T\right)-\pi^{\#}-\left(\tau_{2}-T\right)-\pi^{*}-\tau_{2}-[\right.$ echo $\left.]\right)$ with probe pulses (indicated by ${ }^{*}$ ) and pump pulse (indicated by \#) were used. ${ }^{31,32}$ The lengths of the $\frac{\pi}{2}$ and $\pi$ pulse at the probe frequency are 16 and $32 \mathrm{~ns}$, respectively. The $\pi$ pulse length at the pump frequency was determined by a nutation experiment, usually at either 16 or $20 \mathrm{~ns}$. The frequency difference between the pump pulse and the probe pulse was $80 \mathrm{MHz} .{ }^{33}$ The value of $\tau_{1}$ was 
adjusted to a maximum in the nuclear modulation envelope of deuterium (600 ns), $\mathrm{T}$ was advanced in 20 ns steps. The program DeerAnalysis2013 was used to analyze DEER spectra. ${ }^{34}$ For each sample, the starting, cutoff and dimension for background subtraction are the same in the oxidized and reduced spectra. After background subtraction, least-square fitting was performed using Tikhonov regularization to avoid over-smoothing or artifact peaks.

Restrained Protein docking with DEER distance restraints. The software package HADDOCK 2.1 was used on a local computer cluster for protein docking. ${ }^{35}$ The topology parameters for heme and the iron-sulfur cluster were provided by Prof. Alexandre Bovin and Dr. Angelo Gallo (Center for Magnetic Resonance (CERM), University of Florence) ${ }^{36}$ The procedure used to implement cofactors into HADDOCK and protein docking simulation followed a previous report. ${ }^{37}$ Substrate, ions, water molecules and MTSL were not included explicitly in the docking simulations. ${ }^{38}$ The $\mathrm{P} 450 \mathrm{cam} / \mathrm{Pdx}$ complexes reported were those obtained after rigid-body energy minimization and subsequent semi-flexible refinement with applied DEER distance restraints. For comparison of the P450cam/Pdx complexes obtained by DEER distance restraints and the crystal structure, alignment was performed by Pymol with heme as the reference.

An approach referred to as the rotamer cone model was used to apply DEER distance restraints in HADDOCK docking calculations. This approach considered that the precise placements of spin-label positions are somewhat uncertain due to the unknown MTSL side-chain dihedral angles. Previous studies have shown than uncertainties in spin-label position are orientation dependent and generally lie within approximately \pm 6 A relative to the two $\mathrm{C} \beta$ backbone atoms. ${ }^{39}$ Thus without additional information, the rotamer cone model was used, and DEER derived intermolecular distances were given a $6 \AA$ wide flat-bottomed penalty during docking to account for this uncertainty. This model was initially applied to the open $\mathrm{P} 450 \mathrm{cam}\left(\mathrm{Fe}^{3+}\right) / \mathrm{Pdx}_{\mathrm{ox}}$ complex 
because the actual structure of the complex is known independently by crystallography ${ }^{21}$ and could thus be used as a starting point in the docking simulations. 


\section{RESULTS}

Isothermal titration calorimetry. Shown in Figure 1 are isothermal titration calorimetry (ITC) experiments designed to reveal the interaction thermodynamics between $\mathrm{Pdx}$ and various states of P450cam constrained in different conformations. In panel A, the titration was performed on metyrapone-bound P450cam. This ligand, which binds very tightly to the distal substrate channel by coordinating to the heme is known to induce the closed conformation. ${ }^{13}$ Optical spectra (not shown) did not display any changes in the presence of Pdx indicating that it remains in the closed conformation upon binding Pdx. In panel B, Pdx is titrated into camphor-bound P450cam, and represents the effector induced conformational change from the closed to the open state upon binding Pdx. ${ }^{21,22,28}$ In panel C, the titration utilized substrate-free P450cam, which is known to exist in the open conformation even in the absence of Pdx. ${ }^{40}$ Finally in panel D, Pdx is titrated into P450cam bound to the synthetic ligand Ada-Eg-Dans, which has been shown to constrain the enzyme in a state that is intermediate between open and closed. ${ }^{25}$ Fits to the ITC data in Figure 1 are shown in Table 1, which shows a small but significant increase in affinity between $\mathrm{Pdx}$ and P450cam as the ligand is changed. P450cam bound to metyrapone, which is proposed to remain in the closed state binds to Pdx with a $\mathrm{K}_{\mathrm{d}}$ of $45 \mu \mathrm{M}$. When bound to camphor, which allows for the Pdx induced closed-to-open conversion binds with an affinity of $29 \mu \mathrm{M}$, while substrate-free enzyme, which is already in the open conformation before Pdx binding exhibits a $\mathrm{K}_{\mathrm{d}}$ of $15 \mu \mathrm{M}$. Of significant interest, P450cam bound to the Ada-Eg-Dans synthetic ligand binds to Pdx most tightly of all, with a $\mathrm{K}_{\mathrm{d}}$ of $11 \mu \mathrm{M}$. This ligand has been shown to hold the enzyme in an intermediate conformation between open and closed due to sterically blocking the substrate access channel. ${ }^{25}$ With the exception of metyrapone, each of the Pdx/P450cam binding interactions are enthalpically driven. The results for camphor-bound and camphor-free states are 
in reasonable agreement with recent ITC measurements of Hollingsworth, et al. ${ }^{29}$ Our results show, as proposed, that Pdx binds most strongly to P450cam in an intermediate open state followed by the fully open state of substrate-free enzyme. An additional energy penalty of about $0.4 \mathrm{kcal} / \mathrm{mol}$ is encountered if $\mathrm{Pdx}$ is used to drive the conversion of closed camphor-bound enzyme to the open state. This is thus the energy cost of the effector-induced conversion. Finally, holding the enzyme in the closed conformation as with the metyrapone bound state costs an additional $0.4 \mathrm{kcal} / \mathrm{mol}$ of binding energy. Overall, the ITC data clearly show the expected variation in Pdx binding as the conformational state of P450cam is altered.

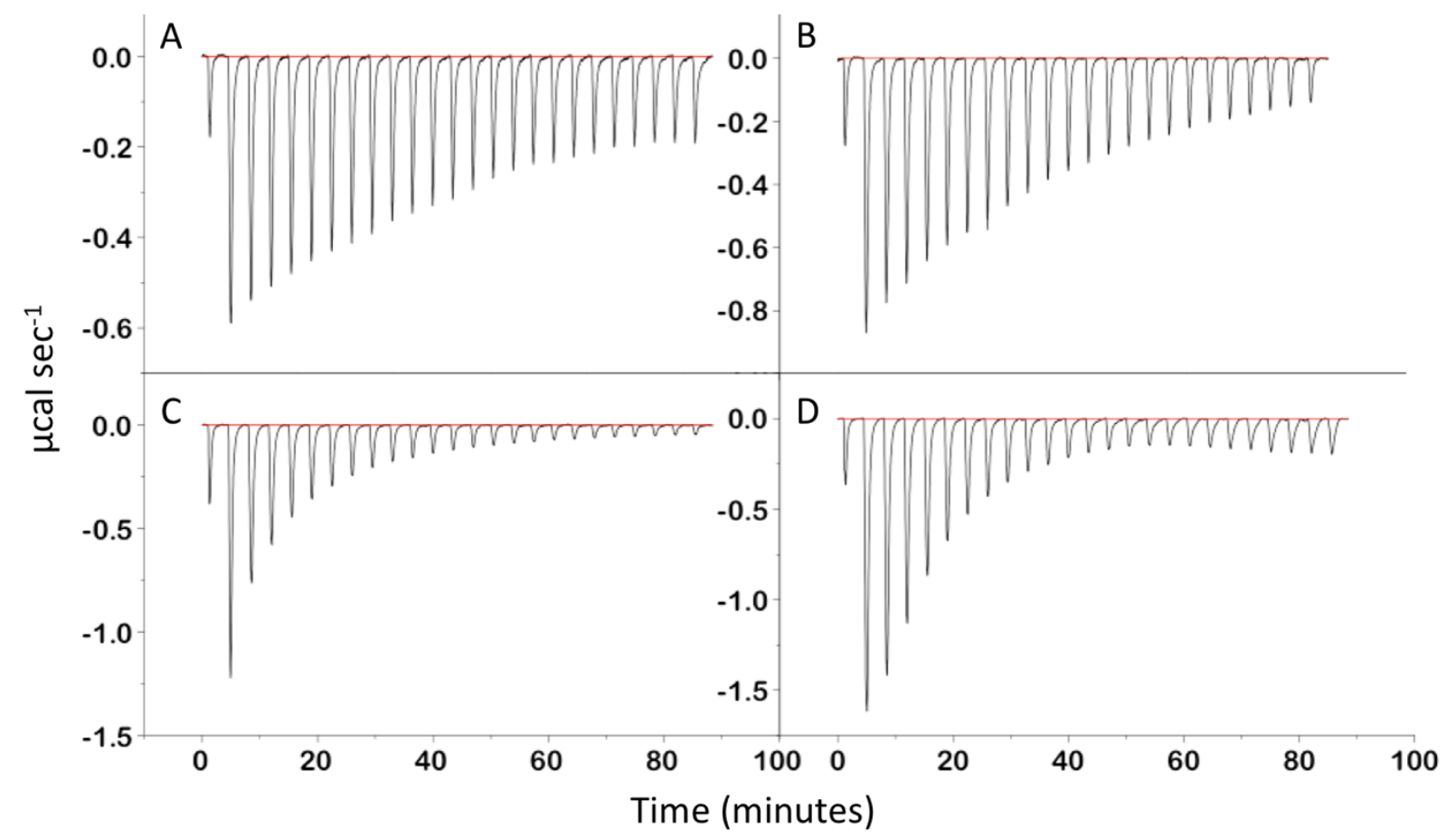

Figure 1. ITC data for the interaction between Pdx and P450cam in various conformational states. Shown are titrations $\mathrm{Pdx}$ into (A) P450cam/metyrapone, which is constrained in the closed conformation, ${ }^{13}$ (B) P450cam/camphor, which converts from the closed to open conformation upon binding $\mathrm{Pdx},{ }^{21,}{ }^{22}$ (C) P450cam (substrate-free), which is in the open 
conformation even in the absence of $\mathrm{Pdx},{ }^{40}$ and (D) P450cam/Ada-EG-Dans, which has been shown to populate a conformation of P450cam that is intermediate between open and closed. ${ }^{25}$

DEER measurements of the Pdx/P450cam complex. To look for structural differences in the Pdx/P450cam interaction between the open and closed forms of P450cam, a series of intermolecular distance measurements were made using DEER. We have shown previously that in the oxidized $\mathrm{P} 450 \mathrm{cam}\left(\mathrm{Fe}^{3+}\right) / \mathrm{Pdx}_{\mathrm{ox}}$ complex, $\mathrm{P} 450$ cam is in the open conformation, while for the $\mathrm{P} 450 \mathrm{cam}\left(\mathrm{Fe}^{2+} \mathrm{CO} \cdot \mathrm{S}\right) / \mathrm{Pdx}_{\mathrm{re}}$ complex, the $\mathrm{P} 450$ cam remains closed due to the ferrous-CO coordination. ${ }^{28}$ This provides a convenient set of conditions to probe the Pdx/P450cam interface in the open and closed states. Twelve intermolecular distances within the Pdx/P450cam complex were prepared by placing one MTSL onto P450cam and another onto Pdx. For P450cam, the labeling sites were S48C, S190C, Q227C or Q272C, while for Pdx, the labeling sites were C73, S29C or E14C. S48C and S190C are at opposite ends of the substrate access channel to provide a longer distance measurement, while the other two positions (Q227C and Q272C) were chosen to triangulate over shorter distances across the complex interface. Each of the 12 complex pairs were labeled, verified by ESI mass spectroscopy (see SI) and DEER samples prepared in the open $\mathrm{P} 450 \mathrm{cam}\left(\mathrm{Fe}^{3+}\right) / \mathrm{Pdx}$ ox or closed $\mathrm{P} 450 \mathrm{cam}\left(\mathrm{Fe}^{2+} \mathrm{CO} \cdot \mathrm{S}\right) / \mathrm{Pdx}_{\mathrm{re}}$ states. The redox state of each sample was verified by UV/Vis and cwEPR (see SI). 


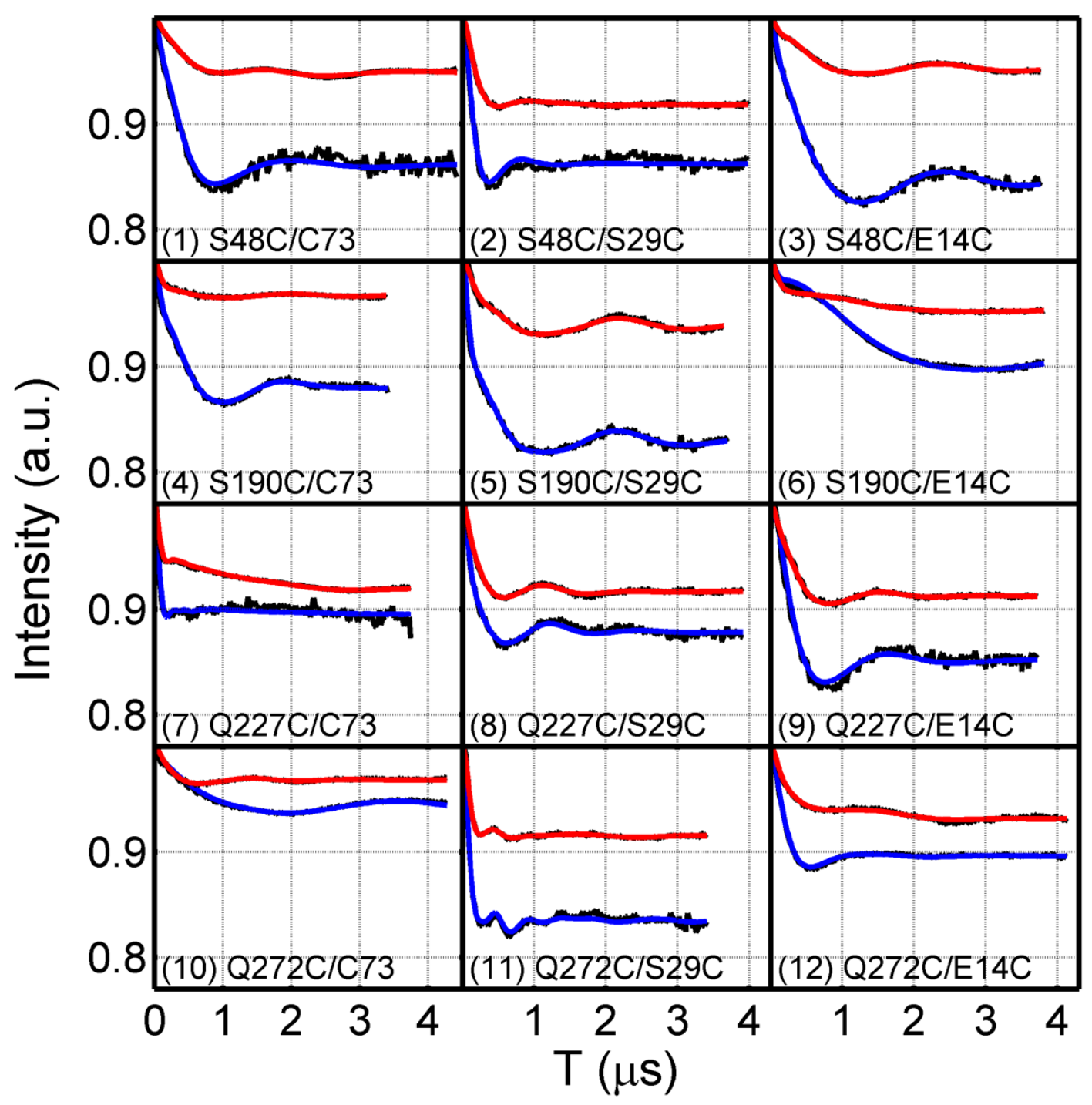

Figure 2. Time-domain DEER spectra for the P450cam/Pdx mutants. The blue and red curves are the fitted DEER curves of $\mathrm{P} 450 \mathrm{cam}\left(\mathrm{Fe}^{3+}\right) / \mathrm{Pdx}_{\mathrm{ox}}$ and $\mathrm{P} 450 \mathrm{cam}\left(\mathrm{Fe}^{2+} \mathrm{CO} \cdot \mathrm{S}\right) / \mathrm{Pdx}$ re, respectively. The black curves are the experimental data. The spin-label positions are indicated by specifying the cysteines introduced into P450cam/Pdx, respectively. 


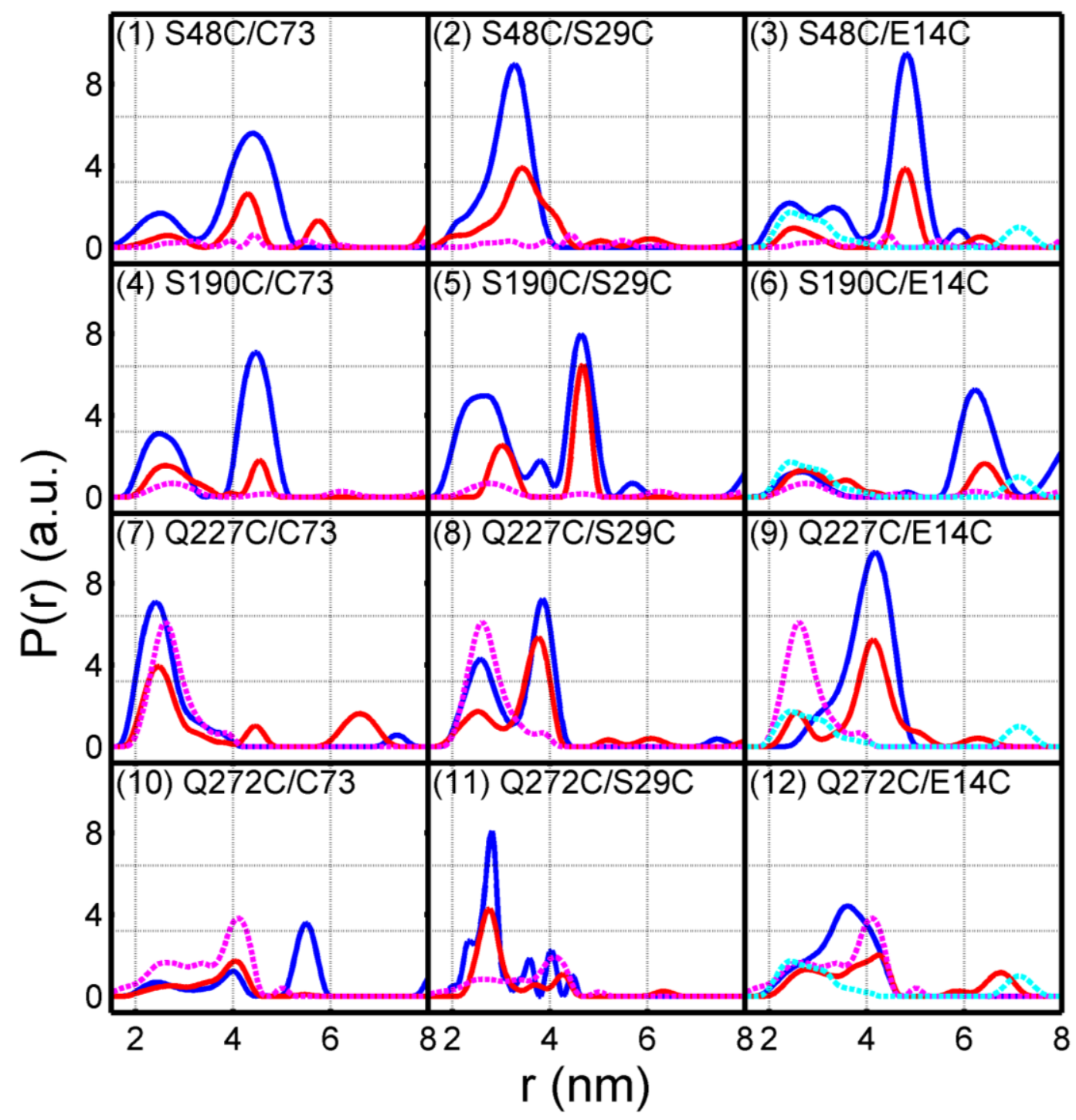

Figure 3. Distance distributions obtained by Tikhonov regularization of the DEER data for the $\mathrm{P} 450 \mathrm{cam} / \mathrm{Pdx}$ complex in the open, oxidized state (blue) and the closed, ferrous- $\mathrm{CO}$ (red) state. The dashed purple lines are the distance modulations from control samples containing singly labeled ferrous-CO P450cam and unlabeled $\mathrm{Pdx}_{\mathrm{re}}$. The dashed cyan curve is a control complex containing unlabeled ferric P450cam and oxidized $\mathrm{Pdx}_{\mathrm{E} 14 \mathrm{C}}$ containing spin-label. These controls were used to identify and eliminate peaks from consideration that may arise from homomolecular interactions. 
For each of the twelve distance pairs, Q-band DEER measurements were made, and the timedomain spectra and distance distributions after Tikhonov regulation are shown in Figures 2 and

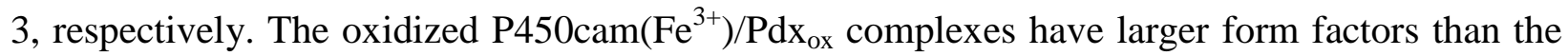
reduced $\mathrm{P} 450$ cam $\left(\mathrm{Fe}^{2+} \mathrm{CO} \cdot \mathrm{S}\right) / \mathrm{Pdx}_{\text {re }}$ complexes. This may be due to the fact that the binding affinity of $\mathrm{Pdx} \mathrm{x}_{\mathrm{ox}}$ for ferric P450cam is approximately $17 \mu \mathrm{M},{ }^{41}$ compared with $26 \mu \mathrm{M}$ for $\mathrm{Pdx} \mathrm{x}_{\mathrm{re}}$ binding to reduced CO-bound P450cam. ${ }^{41-43}$ For some of the spin-labeled pairs of Fig. 3, multiple peaks were observed, making assignments of intermolecular distances ambiguous. The multiple peaks could result from noise or effects of protein aggregation. To identify problematic cases of P450-P450 or Pdx-Pdx interactions, control DEER measurements were made on samples of the complex containing only a single spin-label. These control distance distributions are overlaid on the data of Fig. 3, and identify at least one such case in which no unique distance for the reduced $\mathrm{P} 450 \mathrm{cam}_{\mathrm{Q} 272 \mathrm{C}} / \mathrm{Pdx}_{\mathrm{C} 73}$ could be assigned. Another potential case is with $\mathrm{P}_{450 \mathrm{cam}_{\mathrm{Q} 272}} / \mathrm{Pdx} \mathrm{E}_{\mathrm{E} 4 \mathrm{C}}$. DEER derived distance measurements for the remaining open and closed complexes are listed in Table 2.

One observation about the distance data of Table 2 is immediately clear and unambiguous. Given our previous experience with this system, we estimate the uncertainty for DEER measurements under these conditions to be about $1 \AA$. Each of the measured distances in Table 2 , except one, are at or within this limit when comparing the open and closed complexes. The one exception, for $\mathrm{P} 450 \mathrm{cam}_{\mathrm{Q} 272 \mathrm{C}} / \mathrm{Pdx}_{\mathrm{E} 14 \mathrm{C}}$, could be in error due to the presence of a peak in the singly labeled control mentioned above. Thus, it is clear that whatever the geometry of the solution complex, our DEER measurements show that this complex is the same, within error, for Pdx bound to the open and closed forms of P450cam. 
A docking calculation using the program HADDOCK and including the 12 distance restraints described above was initiated using components of the crystal structure $4 \mathrm{JWS}{ }^{21}$ After rigid-body energy minimization and semi-flexible refinement docking, the structure with the highest score was compared to the oxidized P450cam/Pdx crystal structure, as shown in Figure 4. After alignment of the heme groups of P450cam, the RMSD for the two Pdx molecules was $4.45 \AA$, the center of mass was within $1 \AA$, and the two molecules were related by a 21 degree rotation. As shown in Figure 4, the DEER restrained docked structure maintains the hydrophobic interactions between the $\mathrm{C}$ helix of P450cam and the $\mathrm{C}$ terminus of Pdx. Thus, the DEER restrained HADDOCK docking result is in excellent agreement with the crystallographic structure. $^{44,45}$

A) crystal structure

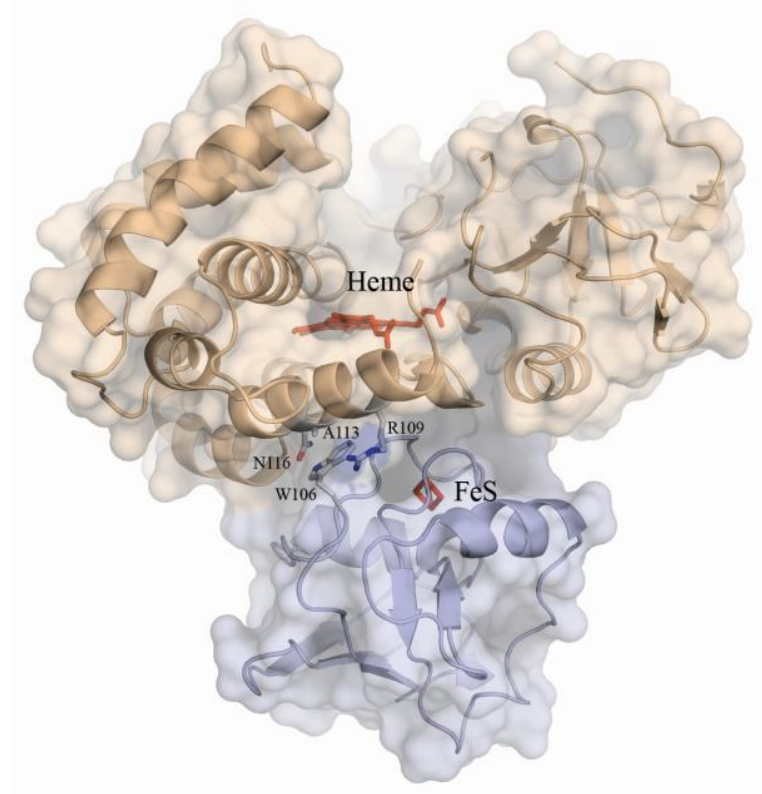

B) DEER restrained open complex

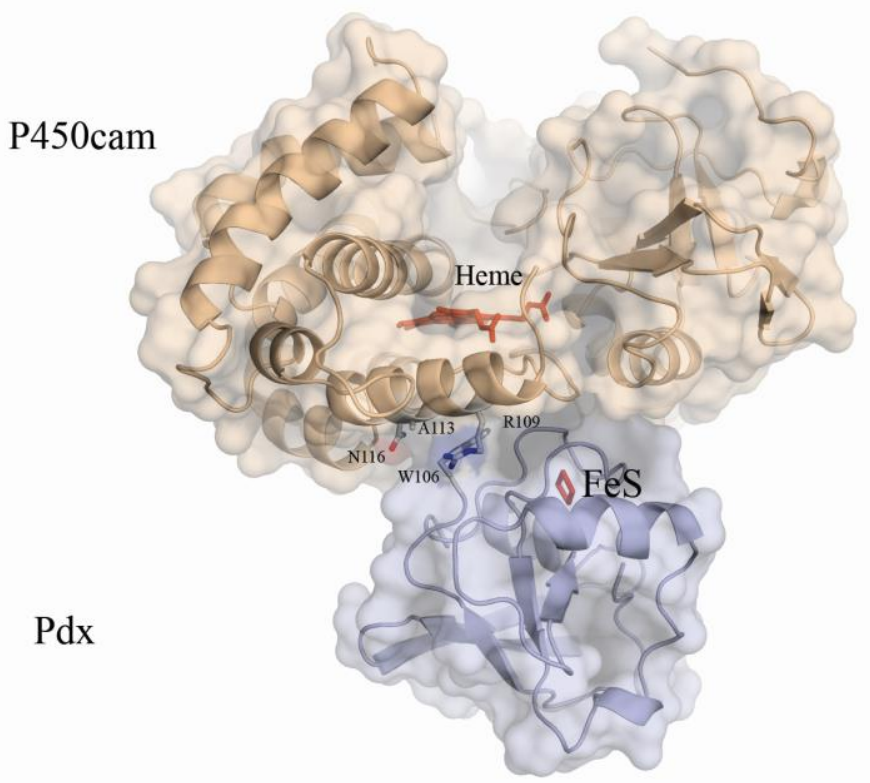

Figure 4. Comparison of the tethered $\mathrm{P} 450 \mathrm{cam} / \mathrm{Pdx}$ complex structure obtained by $\mathrm{X}$-ray crystallography (pdb 4JWS) (A) with the complex obtained following HADDOCK docking 
using DEER distance restraints (B). P450 is shown in tan and Pdx is shown in blue. Specific residues identified in the interface of the crystal structure are labeled.

\section{DISCUSSION}

The effector role of Pdx upon binding P450cam has recently come into significantly improved focus, yet a number of unresolved issues remain. With the proteins in their oxidized state, $\mathrm{Pdx}$ is now generally understood to induce conversion of P450cam to the open state. ${ }^{23-25}$ In the reduced states, where the effector role of $\mathrm{Pdx}$ is most relevant, the current picture is more controversial. Crystal structures of the reduced complex show the enzyme in the open state. ${ }^{21}$ However, crystals of the complex were initially grown in the oxidized state, and the enzyme is known to resist conformational change in the crystal lattice. ${ }^{28}$ DEER studies have shown that the ferrousCO complex bound to either oxidized ${ }^{28}$ or reduced ${ }^{22} \mathrm{Pdx}$ remains closed. Finally, NMR data have been reported that suggest that Pdx binding does not induce conversion to the open state, a result that remains inconsistent with crystallography and DEER studies. ${ }^{26}$ Together, the balance of recent results suggest that $\mathrm{Pdx}$ favors binding to the open form of P450cam when the proteins are oxidized, but its effect on the physiologically relevant ferrous- $\mathrm{O}_{2}$ form remains more uncertain. It is possible that Pdx always favors binding to the open conformation of P450cam and that this is the basis of its effector role. In this view, the inability of Pdx to cause opening of the ferrous-CO complex may result from a specific effect of $\mathrm{CO}$ opposing the conversion to the open state, or it could reflect a true difference in the energetic or dynamic proclivity of the reduced enzyme to convert to the open state.

The observation that Pdx induces the conversion of oxidized P450cam from the closed to the open state requires that the energetics and/or structural interactions between Pdx and P450cam are different for the open and closed conformations. In this work we show that the binding 
thermodynamics of Pdx does indeed differ in the predicted way as the conformation of P450cam is held in different states. However, detailed distance measurements of the complex in solution by DEER show that the complex formed between $\mathrm{Pdx}$ and the open state of the $\mathrm{P} 450$ cam $\left(\mathrm{Fe}^{3+}\right) / \mathrm{Pdx}_{\text {ox }}$ complex is very similar to that observed previously by crystallography. ${ }^{21}$ In addition, Pdx bound to the closed form of P450cam, observed as the $\mathrm{P} 450$ cam $\left(\mathrm{Fe}^{2+} \mathrm{CO} \cdot \mathrm{S}\right) / \mathrm{Pdx}_{\mathrm{ox}}$ complex, is identical within error to that of the open complex. The absolute distances obtained by DEER for individual spin-label pairs across the complex cannot be directly interpreted in terms of protein backbone distances, due to the unknown MTSL sidechain dihedral angles. This was taken into consideration by applying a flat bottomed penalty to the distance restraint energy term in HADDOCK. The docking complex obtained using DEER restraints places Pdx within $4 \AA$ RMSD of that seen in the crystal structure, a result that we consider to be in good agreement. Furthermore, it is likely that most of the MTSL side-chain rotamers will be very similar in the open and closed complexes, unless they are affected by direct steric conflicts. None of our introduced label pairs are near enough to the binding interface to expect such interactions. Thus, the precision of the distance differences observed between open and closed complexes is expected to be very good, and perhaps similar to the estimated $1 \AA$ precision for a particular measurement. The fact that nearly all distance pairs differ by no more than about $1 \AA$ between the open and closed complexes provides strong evidence that, within the error of these MTSL DEER measurements, Pdx binds at the same site for the two forms. It may be useful to extend these studies in the future using bi-functional spin-labels, as we have found that they can give significantly greater distance resolution. ${ }^{27}$ 


\section{CONCLUSIONS}

Our results suggest that in solution, the $\mathrm{Pdx} / \mathrm{P} 450$ cam complex is very similar to that observed in the tethered complex by crystallography. ${ }^{21}$ In addition, the binding site for Pdx appears to be the same in the open and closed states of P450cam. This suggests that ensemble searches of Pdx

for alternative sites on the enzyme surface ${ }^{46}$ do not play a dominant role in recognition and effector function. Our results also show that Pdx binds most tightly to the intermediate and open conformations, and that the energy penalty of binding to the closed conformation is similar to that required to drive the Pdx-induced opening. Thus, Pdx appears to make a single distinct interaction with its binding site on the enzyme in both the closed and open conformations, yet triggers the conformational change through very subtle structural interactions at the binding interface.

\section{ASSOCIATED CONTENT}

\section{Supporting information}

ESI-MS spectra, cwEPR and UV-vis spectra, DEER data analysis details. This material is available free of charge via the Internet at http://pubs.acs.org.

\section{AUTHOR INFORMATION}

\section{Corresponding Author*:}

Telephone: (530) 752-5189. E-mail: dbgoodin@ucdavis.edu

\section{Notes}

The authors declare no conflict of interest. 


\section{ACKNOWLEDGMENTS}

This work was supported by NIH (GM41049 to D.B.G.) and the Department of Energy (DEFG02-09ER16117 to R.D.B.). S.-H.L. thanks the CalEPR center for instrumental and technical support, and Prof. Alexei Stuchebrukhov for access to the computer cluster, and Prof. Alexandre Bovin and Prof. Angelo Gallo for HADDOCK simulation advice. We thank Dr. Young-Tae Lee, Dr. Sang-moon Lee and Mo Zhang for helpful discussions. 


\section{REFERENCES}

[1] Sono, M., Roach, M. P., Coulter, E. D., and Dawson, J. H. (1996) Heme-Containing Oxygenases, Chem Rev 96, 2841-2888.

[2] Denisov, I. G., Makris, T. M., Sligar, S. G., and Schlichting, I. (2005) Structure and Chemistry of Cytochrome P450, Chem. Rev. 105, 2253-2277.

[3] Poulos, T. L. (2014) Heme enzyme structure and function, Chem. Rev. 114, 3919-3962.

[4] Griffin, B. W., and Peterson, J. A. (1972) Camphor binding by Pseudomonas putida cytochrome P-450. Kinetics and thermodynamics of the reaction, Biochemistry 11, 47404746.

[5] Fisher, M. T., and Sligar, S. G. (1987) Temperature jump relaxation kinetics of the P-450cam spin equilibrium, Biochemistry 26, 4797-4803.

[6] Davydov, R., Macdonald, I. D. G., Makris, T. M., and Sligar, S. (1999) EPR and ENDOR of Catalytic Intermediates in Cryoreduced Native and Mutant Oxy-Cytochromes P450 cam: Mutation-Induced Changes in the Proton Delivery System, J. Am. Chem. Soc. 121, 10654-10655.

[7] Schlichting, I., Berendzen, J., Chu, K., Stock, A. M., Maves, S. A., Benson, D. E., Sweet, R. M., Ringe, D., Petsko, G. A., and Sligar, S. G. (2000) The catalytic pathway of cytochrome P450cam at atomic resolution, Science 287, 1615-1622.

[8] Macdonald, I. D. G., Sligar, S. G., Christian, J. F., Unno, M., and Champion, P. M. (1999) Identification of the Fe-O-O Bending Mode in Oxycytochrome P450cam by Resonance Raman Spectroscopy, J. Am. Chem. Soc. 121, 376-380.

[9] Nagano, S., and Poulos, T. L. (2005) Crystallographic study on the dioxygen complex of wild-type and mutant cytochrome P450cam. Implications for the dioxygen activation mechanism, J. Biol. Chem. 280, 31659-31663.

[10] Davydov, R., Dawson, J. H., Perera, R., and Hoffman, B. M. (2013) The use of deuterated camphor as a substrate in (1)H ENDOR studies of hydroxylation by cryoreduced oxy P450cam provides new evidence of the involvement of compound I, Biochemistry 52, 667-671.

[11] Yosca, T. H., and Green, M. T. (2016) Preparation of Compound I in P450cam: The Prototypical P450, Isr. J. Chem. 56, 834-840.

[12] Poulos, T. L., Finzel, B. C., Gunsalus, I. C., Wagner, G. C., and Kraut, J. (1985) The 2.6.ANG. crystal structure of Pseudomonas putida cytochrome P-450, J. Biol. Chem 260, 16122-16130.

[13] Poulos, T. L., and Howard, A. J. (1987) Crystal structures of metyrapone- and phenylimidazole-inhibited complexes of cytochrome P-450cam, Biochemistry 26, 81658174.

[14] Poulos, T. L., Finzel, B. C., and Howard, A. J. (1987) High resolution crystal structure of cytochrome P450cam, J. Mol. Biol. 195, 687-700.

[15] Sevrioukova, I. F., and Poulos, T. L. (2011) Structural biology of redox partner interactions in P450cam monooxygenase: a fresh look at an old system, Arch. Biochem. Biophys. 507, 66-74.

[16] Lipscomb, J. D. (1980) Electron paramagnetic resonance detectable states of cytochrome P450cam, Biochemistry 19, 3590-3599.

[17] Sjodin, T., Christian, J. F., Macdonald, I. D. G., Davydov, R., Unno, M., Sligar, S. G., Hoffman, B. M., and Champion, P. M. (2001) Resonance Raman and EPR Investigations 
of the D251N Oxycytochrome P450cam/Putidaredoxin Complex, Biochemistry 40, 68526859.

[18] Unno, M., Christian, J. F., Sjodin, T., Benson, D. E., Macdonald, I. D. G., Sligar, S. G., and Champion, P. M. (2002) Complex formation of cytochrome P450cam with putidaredoxin: evidence for protein-specific interactions involving the proximal thiolate ligand, J. Biol. Chem. 277, 2547-2553.

[19] Tosha, T., Yoshioka, S., Ishimori, K., and Morishima, I. (2004) L358P mutation on cytochrome P450cam simulates structural changes upon putidaredoxin binding: the structural changes trigger electron transfer to oxy-P450cam from electron donors, J. Biol. Chem. 279, 42836-42843.

[20] Nagano, S., Tosha, T., Ishimori, K., Morishima, I., and Poulos, T. L. (2004) Crystal structure of the cytochrome p450cam mutant that exhibits the same spectral perturbations induced by putidaredoxin binding, J. Biol. Chem. 279, 42844-42849.

[21] Tripathi, S., Li, H., and Poulos, T. L. (2013) Structural basis for effector control and redox partner recognition in cytochrome P450, Science 340, 1227-1230.

[22] Myers, W. K., Lee, Y. T., Britt, R. D., and Goodin, D. B. (2013) The conformation of p450cam in complex with putidaredoxin is dependent on oxidation state, J. Am. Chem. Soc. 135, 11732-11735.

[23] Lee, Y.-T., Wilson, R. F., Rupniewski, I., and Goodin, D. B. (2010) P450cam visits an open conformation in the absence of substrate, Biochemistry 49, 3412-3419.

[24] Stoll, S., Lee, Y. T., Zhang, M., Wilson, R., Britt, R. D., and Goodin, D. B. (2012) Double electron-electron resonance shows cytochrome P450cam undergoes a conformational change in solution upon binding substrate, Proc. Natl. Acad. Sci. U.S.A. 109, 1288812893.

[25] Lee, Y. T., Glazer, E. C., Wilson, R. F., Stout, C. D., and Goodin, D. B. (2011) Three clusters of conformational states in p450cam reveal a multistep pathway for closing of the substrate access channel, Biochemistry 50, 693-703.

[26] Skinner, S. P., Liu, W. M., Hiruma, Y., Timmer, M., Blok, A., Hass, M. A., and Ubbink, M. (2015) Delicate conformational balance of the redox enzyme cytochrome P450cam, Proc. Natl. Acad. Sci. U.S.A. 112, 9022-9027.

[27] Hiruma, Y., Hass, M. A., Kikui, Y., Liu, W. M., Olmez, B., Skinner, S. P., Blok, A., Kloosterman, A., Koteishi, H., Lohr, F., Schwalbe, H., Nojiri, M., and Ubbink, M. (2013) The structure of the cytochrome p450cam-putidaredoxin complex determined by paramagnetic NMR spectroscopy and crystallography, J. Mol. Biol. 425, 4353-4365.

[28] Liou, S. H., Mahomed, M., Lee, Y. T., and Goodin, D. B. (2016) Effector Roles of Putidaredoxin on Cytochrome P450cam Conformational States, J. Am. Chem. Soc. 138, 10163-10172.

[29] Hollingsworth, S. A., Batabyal, D., Nguyen, B. D., and Poulos, T. L. (2016) Conformational selectivity in cytochrome $\mathrm{P} 450$ redox partner interactions, Proc. Natl. Acad. Sci. USA $113,8723-8728$.

[30] Sevrioukova, I. F., Garcia, C., Li, H., Bhaskar, B., and Poulos, T. L. (2003) Crystal structure of putidaredoxin, the $[2 \mathrm{Fe}-2 \mathrm{~S}]$ component of the $\mathrm{P} 450 \mathrm{cam}$ monooxygenase system from Pseudomonas putida, J. Mol. Bio. 333, 377-392.

[31] Pannier, M., Veit, S., Godt, A., Jeschke, G., and Spiess, H. W. (2000) Dead-time free measurement of dipole-dipole interactions between electron spins, J Magn Reson 142, 331-340. 
[32] Jeschke, G. (2012) DEER distance measurements on proteins, Annu. Rev. Phys. Chem. 63, 419-446.

[33] Polyhach, Y., Bordignon, E., Tschaggelar, R., Gandra, S., Godt, A., and Jeschke, G. (2012) High sensitivity and versatility of the DEER experiment on nitroxide radical pairs at Qband frequencies, Phys. Chem. Chem. Phys. 14, 10762-10773.

[34] Jeschke, G., Chechik, V., Ionita, P., Godt, A., Zimmermann, H., Banham, J., Timmel, C. R., Hilger, D., Jung, H. (2006) DeerAnalysis2006---a comprehensive software package for analyzing pulsed ELDOR data, Appl. Magn. Reson. 30, 473-498.

[35] Dominguez, C., Boelens, R., and Bonvin, A. M. J. J. (2003) HADDOCK: A Protein-Protein Docking Approach Based on Biochemical or Biophysical Information, J. Am. Chem. Soc. 125, 1731-1737.

[36] Bancia, L., Bertini, I., Calderone, V., Ciofi-Baffoni, S., Giachetti, A., Jaiswal, D., Mikolajczyk, M., Piccioliand, M., and Winkelmann, J. (2013) Molecular view of an electron transfer process essential for iron-sulfur protein biogenesis, Proc. Natl. Acad. Sci. U S A 110, 7136-7141.

[37] Clair, S. V. L. (2013) Characterization of the membrane-bound full-length complex between cytochrome b5 and cytochrome P450 2B4, In Deparment of Chemistry, University of Michigan.

[38] Hagelueken, G., Abdullin, D., and Schiemann, O. (2015) mtss1Suite: Probing Biomolecular Conformation by Spin-Labeling Studies, Methods Enzymol. 563, 595-622.

[39] Hirst, S. J., Alexander, N., McHaourab, H. S., and Meiler, J. (2011) RosettaEPR: an integrated tool for protein structure determination from sparse EPR data, J. Struct. Biol. 173, 506-514.

[40] Lee, Y. T., Wilson, R. F., Rupniewski, I., and Goodin, D. B. (2010) P450cam visits an open conformation in the absence of substrate, Biochemistry 49, 3412-3419.

[41] Zhang, W., Pochapsky, S. S., Pochapsky, T. C., and Jain, N. U. (2008) Solution NMR structure of putidaredoxin-cytochrome P450cam complex via a combined residual dipolar coupling-spin labeling approach suggests a role for Trp106 of putidaredoxin in complex formation, J. Mol. Bio. 384, 349-363.

[42] Rui, R. L., Pochapsky, S., and Pochapsky, T. (2006) Comparison of the complexes formed by cytochrome P450cam with cytochrome b5 and putidaredoxin, two effectors of camphor hydroxylase activity, Biochemistry 45, 3887-3897.

[43] Pochapsky, S., Pochapsky, T., and Wei, J. Y. (2003) A model for effector activity in a highly specific biological electron transfer complex: the cytochrome P450(cam)putidaredoxin couple, Biochemistry 42, 5649-5656.

[44] Bhatnagar, J., Freed, J. H., and Crane, B. R. (2007) Rigid Body Refinement of Protein Complexes with Long- Range Distance Restraints from Pulsed Dipolar ESR, Methods Enzymol. 423, 117-133.

[45] Smith, J. A., Edwards, S. J., Moth, C. W., and Lybrand, T. P. (2013) TagDock: an efficient rigid body docking algorithm for oligomeric protein complex model construction and experiment planning, Biochemistry 52, 5577-5584.

[46] Andrałojc, W., Hiruma, Y., Liu, W.-M., Ravera, E., Nojiri, M., Parigi, G., Luchinat, C., and Ubbink, M. (2017) Identification of productive and futile encounters in an electron transfer protein complex, Proc. Natl. Acad. Sci. U S A 114, 1840-1847. 
Table 1. ITC parameters for Pdx binding to P450cam in different ligand-enforced conformations.

\begin{tabular}{c|c|c|c|c|c}
\hline P450 ligand & $\mathrm{N}$ & $\begin{array}{c}\mathrm{K}_{\mathrm{d}} \\
(\mu \mathrm{M})\end{array}$ & $\begin{array}{c}\Delta \mathrm{G} \\
(\mathrm{kcal} / \mathrm{mol})\end{array}$ & $\begin{array}{c}\Delta \mathrm{H} \\
(\mathrm{kcal} / \mathrm{mol})\end{array}$ & $\begin{array}{c}-\mathrm{T} \Delta \mathrm{S} \\
(\mathrm{kcal} / \mathrm{mol})\end{array}$ \\
\hline Metyrapone & $1.4(8)$ & $45.3(7)$ & $-5.93(4)$ & $-1.9(2)$ & $-4.0(1)$ \\
\hline Camphor & $1.0(1)$ & $29.0(8)$ & $-6.19(7)$ & $-6.5(2)$ & $0.33(2)$ \\
\hline Substrate-free & $0.8(1)$ & $15.2(8)$ & $-6.6(1)$ & $-9.3(3)$ & $2.7(3)$ \\
\hline Ada-Eg-Dans & $1.0(2)$ & $10.8(7)$ & $-6.8(2)$ & $-9.3(7)$ & $2.5(7)$ \\
\hline
\end{tabular}

Table 2. DEER derived distances $(\AA)$ for the open P450cam $\left(\mathrm{Fe}^{3+}\right) / \mathrm{Pdx}_{\text {ox }}$ complex (blue) and the closed $\mathrm{P} 450 \mathrm{cam}\left(\mathrm{Fe}^{2+} \mathrm{CO} \cdot \mathrm{S}\right) / \mathrm{Pdx}_{\mathrm{re}}$ complex (red).

\begin{tabular}{c|c|c|c}
\hline & C73 Pdx & S29C Pdx & E14C Pdx \\
\hline S48C P450cam & $44 / 43$ & $\mathbf{3 3 / 3 4}$ & $\mathbf{4 8 / 4 8}$ \\
\hline S190C P450cam & $45 / 46$ & $\mathbf{4 6 / 4 7}$ & $\mathbf{6 2 / 6 4}$ \\
\hline Q227C P450cam & $\mathbf{2 4 / 2 5}$ & $\mathbf{3 9 / 3 8}$ & $\mathbf{4 2 / 4 1}$ \\
\hline Q272C P450cam & $55 /-$ & $\mathbf{2 8 / 2 8}$ & $\mathbf{3 6 / 4 3}$ \\
\hline
\end{tabular}


TOC Figure.

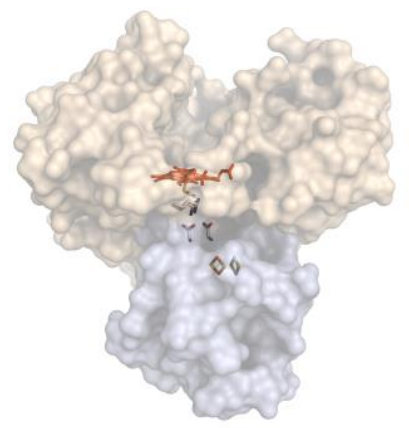

